\title{
The geriatrician remains responsible for appropriate pharmacotherapy
}

\author{
Timo E. Strandberg ${ }^{1,2,3} \cdot$ Riitta Antikainen $^{1,2,3} \cdot$ Risto Huupponen $^{1,2,3} \cdot$ Jouko Laurila $^{1,2,3} \cdot$ Hanna-Maria Roitto $^{1,2,3}$
}

Received: 1 December 2021 / Accepted: 22 December 2021 / Published online: 19 January 2022

(c) The Author(s), under exclusive licence to European Geriatric Medicine Society 2022

\section{Dear Editor,}

In their Editorial "The role of pharmacists in geriatric teams: the time is now" Delgado-Silveira and Bermejo-Vicedo discuss geriatric pharmacotherapy from the viewpoint of pharmacists [1]. In the following, we would like to add some further comments on this discussion by reminding that a geriatrician must have adequate skills to be responsible also for the drug treatment of one's patients. Furthermore, resources in health care are scarce relative to needs, and knowledge on cost-effectiveness of interventions must be established before their adoption into everyday practice.

Assessment of appropriate drug treatment and medication list is certainly one of the main tasks in the treatment of a geriatric patient and a vital part of the comprehensive geriatric assessment. Experts in this field, like pharmacists, are definitely needed in a geriatric team. We are, however, concerned about the possibility that routine involvement of pharmacists may lead physicians to "outsource" medication issues from their expertise. A physician is nevertheless responsible for the totality of care including the appropriateness of pharmacotherapy.

In our view, pharmacists may have the greatest impact on rational pharmacotherapy by resolving complex questions not easily handled during normal patient visits. Polypharmacy is common in older adults and verification of drugs actually taken, complex drug interactions and medication errors are examples of such questions.

This comment refers to the article available online at https://doi. org/10.1007/s41999-021-00501-8.

Timo E. Strandberg

timo.strandberg@oulu.fi

1 University of Helsinki and Helsinki University Hospital (TES, HMR), Haartmaninkatu 4, PO Box 340, 00029 Helsinki, Finland

2 Center for Life Course Health Research (TES, RA, JL), University of Oulu, Oulu, Finland

3 University of Turku (RH), Turku, Finland
In addition, we think that in busy clinical work physicians must have sufficient expertise to be able to handle common medication issues. Consulting routinely other professionals is too time-consuming and may not lead to better results. When checking for drug interactions, adjusting drug dosing in renal failure or estimating medication burden physicians may rely on user-friendly digital tools increasingly available in many countries. This trend should be propagated.

In conclusion, while we warmly welcome pharmacists in the geriatric team, this must not blur the role of the physician who is responsible for the whole picture, including appropriate drug treatment. We cannot afford physicians to lose their interest in medication reviews and the potential benefits and harms they include. Comprehensive geriatric assessment is compromised, if indication-based prescribing and adequate deprescribing skills of physicians are fading.

\section{Declarations}

Conflict of interest TES, RA, JL and HMR are geriatricians. RH is a physician and clinical pharmacologist.

Ethical approval Not applicable.

Informed consent Not applicable.

\section{Reference}

1. Delgado-Silveira E, Bermejo-Vicedo T (2021) The role of pharmacists in geriatric teams: the time is now. Eur Geriatr Med 12(6):1119-1121. https://doi.org/10.1007/s41999-021-00501-8

Publisher's Note Springer Nature remains neutral with regard to jurisdictional claims in published maps and institutional affiliations. 